\title{
Ueber die Anzahl reeller Normalen, welche von einem Punkte an ein Ellipsoid gezogen werden können.
}

(Von Herrn F. Joachimsthal zu Breslau.)

§. 1 .

$\mathrm{E}_{\mathrm{S} \text { sei }} \frac{x^{2}}{a}+\frac{y^{2}}{b}+\frac{z^{2}}{c}=1$ die Gleichung des Ellipsoides, $(\xi, \eta, \zeta)$ der gegebene Punkt; für den Fusspunkt einer durch $(\xi, \eta, \zeta)$ gehenden Normale gelten die Gleichungen

$$
\text { (1.) } \frac{\xi-x}{\frac{x}{a}}=\frac{\eta-y}{\frac{y}{b}}=\frac{\zeta-z}{\frac{z}{c}} \text {. }
$$

Setzt man diese Brüche $=-u$, so erhält man

$$
\text { (2.) } \quad x=\frac{a \xi}{a-u}, \quad y=\frac{b \eta}{b-u}, \quad z=\frac{c \xi}{c-u},
$$

und zur Bestimmung von $u$ die Gleichung sechsten Grades

$$
\frac{a \xi^{2}}{(a-u)^{2}}+\frac{b \eta^{2}}{(b-u)^{2}}+\frac{c \zeta^{2}}{(c-u)^{2}}=1
$$

Wie sich aus (1.) ergiebt, ist $u$ das Product der Entfernungen des Punktes $(\xi, \eta, \zeta)$ und des Anfangspunktes von der das Ellipsoid in $(x, y, z)$ berührenden Ebene, dieses Product positiv oder negativ genommen, je nachdem beide Punkte auf derselben Seite der Ebene liegen oder nicht. Für den vorliegenden Zweck ist es angemessen in (3) statt $\xi, \eta, \zeta$ drei andere Constante einzuführen *).

*) Für das ebene Problem, über welches ich hier einige Bemerkungen einschalte, dürfte die analoge Gleichung $\frac{a \xi^{2}}{(a-u)^{2}}+\frac{b \eta^{2}}{(b-u)^{2}}=1$ die bequemste und am meisten symmetrische Behandlung der Aufgabe gestatten. Setzt man ihre linke Seite $=f(u)$, so ist für den Fall der Ellipse $(a>0, b>0, a>b) f(u)$ stets positiv, verschwindet nur für $u= \pm \infty$, und wird $=\infty$ für $u=b$ oder $=a ; f^{\prime}(u)=2\left\{\frac{a \xi^{2}}{(a-u)^{3}}+\frac{b \eta^{2}}{(b-u)^{3}}\right\}$ verschwindet nur ein Mal für den Werth $u_{1}$, der zwischen $b$ und $a$ liegt, und durch die Gleichung $\frac{a-u_{1}}{b-u_{1}}=-\left(\frac{a \xi^{2}}{b \eta^{2}}\right)^{\frac{t}{t}}$ gegeben ist. Der zugehörige Werth von $f\left(u_{1}\right)$ ist 
Die Ǵleichung (3.) hat nothwendig zwei reelle Wurzeln, von denen, wenn wir, wie durchweg, $a>b>c$ annehmen, eine zwischen $-\infty$ und $c$, die andere zwischen $a$ und $+\infty$ liegt. Die eine dieser Wurzeln sei bekannt $=v$, und die Coordinaten des Fusspunktes der ihr entsprechenden Normale

demnach ein Minimum $=\frac{\left(a^{\frac{1}{3}} \xi^{\frac{2}{3}}+b^{\frac{1}{3}} \eta^{\frac{2}{3}}\right)^{3}}{(a-b)^{2}}$ und $f(u)$ hat folgenden Gang:

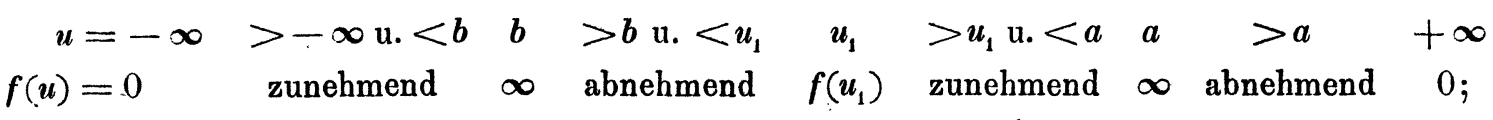
es erreicht daher den Werth eins, je nachdem $f\left(u_{1}\right) \lesseqgtr 1$, vier-, drei- oder zweimal. Für die Hyperbel sind geringe Modificationen des Verfahrens nöthig.

Hat man sich aber auf irgend eine Weise überzeugt, dass die Gleichung, von welcher man das Problem abhängig macht, zwei reelle Wurzeln besitzt, so kann man eine Betrachtung anwenden, die für alle biquadratischen Gleichungen gilt, die diese Eigenschaft besitzen. Sind nämlich $\alpha, \beta, \gamma, \delta$ die Wurzeln einer solchen, so reicht, und das ist das Characteristische dieser Gleichungen, das Vorzeichen der einzigen symmetrischen Function

$$
\Delta=(\alpha-\beta)^{2}(\alpha-\gamma)^{2}(\alpha-\delta)^{2}(\beta-\gamma)^{2}(\beta-\delta)^{2}(\gamma-\delta)^{2}
$$

hin, um zu entscheiden, ob die Gleichung vier oder nur zwei reelle Wurzeln hat, im ersten Falle ist $\Delta$ positiv, im zweiten sind, wenn nur $\alpha$ und $\beta$ reell,

$$
(\alpha-\beta)^{2},(\alpha-\gamma)(\alpha-\delta),(\beta-\gamma)(\beta-\delta)
$$

positiv, dagegen $(\gamma-\delta)^{2}$ negativ, also auch $\Delta$. Da ausserdem $\Delta=0$ die Bedingung zweier gleichen Wurzeln der biquadratischen Gleichung ist, und im Normalenproblem die Coordinaten eines jeden Punktes der Evqluten der Gleichung vierten Grades zwei zusammenfallende Wurzeln verschaffen, so sieht man a priori, dass wenn $\boldsymbol{E}=0$ die rationale Gleichung der Evoluten ist, $E$ zum wenigsten ein Factor von $\Delta$ sein muss. Behufs einer genaueren Vergleichung muss man $\Delta$ als Function der Coefficienten kennen; nach Cayley's schöner Darstellung ist für die Gleichung:

$$
a x^{4}+4 b x^{3}+6 c x^{2}+4 d x+e=0, a^{5} \Delta=J^{3}-27 J_{1}^{2},
$$

wo

$$
J=a e-4 b d+3 c^{2} ; \quad J_{1}=a c e-a d^{2}-e b^{2}-2 c^{3}+2 b c d .
$$

Eine dritte hieher gehörende Betrachtung ist folgende. Sind $U=0, U_{i}=0$ die Gleichungen zweier Kegelschnitte, so ist der Factor $\lambda$, welcher $U+\lambda U_{1}$ in lineare Factoren zerlegbar macht, durch eine kubische Gleichung gegeben, die im Falle von vier reellen Durchschnitten beider Curven drei reelle Wurzeln hat, aber nur eine, wenn die Curven sich in zwei reellen Punkten oder gar nicht schneiden. Weiss man demnach anderweitig, dass die Curven wenigstens zwei reelle Punkte gemein haben, (so z. B. im Normalenproblem der gegebene Kegelschnitt und die Hülfshyperbel), so ist die kubische Gleichung entscheidend.

Die bekānnte Aufgabe „durch einen Punkt eine Grade zu ziehen, deren von zwei schiefwinkligen Axen begrenztes Stück eine gegebene Länge hat" bietet mit dem ebenen Normalenprobleme sehr viele Analogieen dar; namentlich aber ergeben sich die Realitätsbedingungen durch die zuletzt angeführten Methoden, weil die Aufgabe unter ihren vier Lösungen ebenfalls immer zwei reelle hat. 
$=x_{0}, y_{0}, z_{0}$, so erhält man aus (2.) die Gleichungen

$$
\text { (4.) } \xi=\frac{x_{0}(a-v)}{a}, \quad \eta=\frac{y_{0}(b-v)}{b}, \zeta=\frac{z_{0}(c-v)}{c},
$$

und da $\left(x_{0}, y_{0}, z_{0}\right)$ ein Punkt der Fläche, so lassen sich bekanntlich zwei reelle Grössen $\alpha$ und $\beta$ einführen, von denen die grössere $\alpha$ zwischen $a$ und $b$, die kleinere $\beta$ zwischen $b$ und $c$ liegt, so dass

$$
\left(4^{*} .\right) \quad x_{0}^{2}=\frac{a(a-\alpha)(a-\beta)}{(a-b)(a-c)}, \quad y_{0}^{2}=\frac{b(b-\alpha)(b-\beta)}{(b-a)(b-c)}, \quad z_{0}^{2}=\frac{c(c-\alpha)(c-\beta)}{(c-a)(c-b)} .
$$

Mit Hülfe von (4.) und $\left(4^{*}\right.$.) verwandelt sich (3.) in

$$
\frac{(a-\alpha)(a-\beta)}{(a-b)(a-c)} \frac{(a-v)^{2}}{(a-u)^{2}}+\frac{(b-a)(b-\beta)}{(b-a)(b-c)} \frac{(b-v)^{2}}{(b-u)^{2}}+\frac{(c-a)(c-\beta)}{(c-a)(c-b)} \frac{(c-v)^{2}}{(c-u)^{2}}=1 .
$$

Von den Constanten $\alpha, \beta, v$, welche die ursprünglichen $\xi, \eta, \zeta$ ersetzen, bestimmen $\alpha, \beta$ den Fusspunkt einer durch $(\xi, \eta, \zeta)$ gehenden Normale, und $v$ die Lage des Punktes auf derselben, indem, wenn $\pi$ das Loth vom Mittelpunkte der Fläche auf die Tangentialebene ịm Punkte $(\alpha, \beta)$ des Ellipsoides bezeichnet, $\frac{v}{\pi}$ die auf der Normale gemessene Entfernung des Punktes $(\xi, \eta, \zeta)$ vom Fusspunkte bedeutet, die Richtung nach dem Inneren des Ellipsoides als positiv angenommen. Für $\alpha$ und $\beta$ gelten die Bedingungén $a>\alpha>b>\beta>c$, so jedoch, dass das Zusammenfallen von $\alpha$ und $\beta$ mit einem der Grenzwerthe ausgeschlossen bleibe, $d$. h. dass die als gegeben angesehene Normale in keinem der Hauptschnitte liege, ein Fall, der besonders erledigt werden wird. Die Grösse $v$ kann jeden beliebigen Werth haben.

Um aus (5.) die Wurzel $u=v$ fortzuschaffen, schreiben wir

und erhalten

$$
a-v=a-u+u-v, \text { u. s. w., }
$$

$\Sigma \frac{(a-\alpha)(a-\beta)}{(a-b)(a-c)}+2(u-v) \sum \frac{(a-\alpha)(a-\beta)}{(a-b)(a-c)} \frac{1}{a-u}+(u-v)^{2} \Sigma \frac{(a-\alpha)(a-\beta)}{(a-b)(a-c)} \frac{1}{(a-u)^{2}}=1$.

Die erste Summe ist $=1$, die zweite $=\frac{(\alpha-u)(\beta-u)}{(a-u)(b-u)(c-u)}$, und wenn dieser Ausdruck mit $f(u)$ bezeichnet wird, die dritte $=f^{\prime}(u)$. Die vorhergehende Gleichung geht somit in

$$
(u-v)\left\{2 f(u)+(u-v) f^{\prime}(u)\right\}={ }^{\prime} 0
$$

über; oder mit Fortlassung des Factors $u-v$ in

$$
\frac{2}{u-v}=\frac{1}{u-a}+\frac{1}{u-b}+\frac{1}{u-c}-\frac{1}{u-\alpha}-\frac{1}{u-\beta} \text {. }
$$

Journal für Mathematik Bd. LIX. Heft 2. 


\section{Joachimsthal, Ansahl reeller Normalen von einem Punte an ein Ellipsoid.}

Diese Gleichung fünften Grades bildet den Ausgangspunkt gegenwärtiger Untersuchung.

\section{2.}

Es handelt sich darum für jeden Werth von $v$ die Anzahl der reellen Wurzeln von (6.) zu bestimmen. Nun ergiebt sich aus (6.) zu irgend einem reellen Werthe von $u$ durch eine Gleichung ersten Grades ein reeller Werth von $v$. Lasst man $u$ alle Werthe von $-\infty$ bis $+\infty$ durchlaufen, und bekommt hierbei $v$ einen bestimmten Werth $v_{1} n$ mal, so wird umgekehrt (6.), wenn man darin $v_{0}$ statt $v$ einsetzt, $n$ reelle Wurzeln $u$ haben. Es ist daher der Gang von $v$ als einer durch (6.) definirten Function von $u$ zu untersuchen. Die rechte Seite von (6.) $\frac{1}{u-a}-\frac{1}{u-a}+\frac{1}{u-b}-\frac{1}{u-\beta}+\frac{1}{u-c}$ lässt sich schreiben

$$
\text { (7.) } \frac{1}{u-a}-\frac{\alpha-b}{(u-\alpha)(u-b)}-\frac{\beta-c}{(u-\beta)(u-c)}
$$

und

$$
\text { (7. *) } \frac{a-\alpha}{(u-a)(u-\alpha)}+\frac{b-\beta}{(u-b)(u-\beta)}+\frac{1}{u-c} \text {. }
$$

'In Folge der Ungleichungen $a>\alpha>b>\beta>c$ wird, wenn man den Raum von $-\infty$ bis $+\infty$ in die sechs Intervalle theilt

$-\infty$ bis $c, \quad c$ bis $\beta, \quad \beta$ bis $b, \quad b$ bis $\alpha, \quad \alpha$ bis $a, \quad a$ bis $+\infty$, für das erste, dritte und fünfte Intervall (7.) eine Summe negativer Glieder, und (7. *) für die drei anderen eine Summe positiver Glieder sein. Die linke Seite von (6.) verschwindet demnach für kein endliches reelles $u$. Dasselbe findet daher auch, wenn man aus (6.) den Werth von $v$ ableitet,

$$
\text { (8.) } \quad 0=u-2 \frac{(u-a)(u-\alpha)(u-b)(u-\beta)(u-c)}{W}=\frac{U}{W} \text {, }
$$

wo $U$ vom fünften, $W$ vom vierten Grade ist, für den Nenner $W$ statt, der, weil er mit $+u^{4}$ beginnt, stets positiv bleibt; somit ist $v$ eine continuirliche Function von $u$. Vermittelst (8.) gewinnen wir den ersten Ueberblick uber ihren Gang

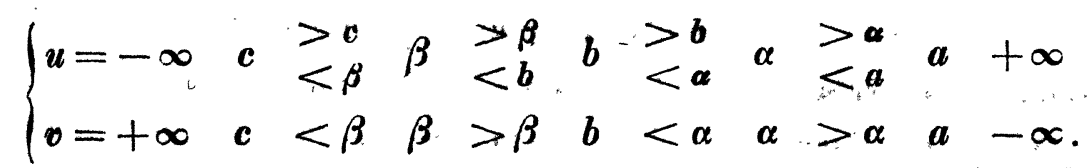

Die weitere Kenntniss des Ganges von' verlangt die Bestimmung seiner 
Maxima und Minima. Nun ist zufolge (8.) $\frac{d v}{d u}=-\frac{\varphi(u)}{W^{2}}$ (wo $\varphi(u)$ einen mit $+u^{8}$ beginnenden Ausdruck achten Grades bedeutet), also ebenfalls eine continuirliche Function von $u$, und $v$ wird nur für die Wurzeln von $\varphi(u)=0$ ein Maximum oder Minimum; da nun $\frac{d^{2} v}{d u^{2}}=-\frac{W \mathscr{q}^{\prime}(u)-2 W^{\prime} \varphi(u)}{W^{3}}$ für die Wurzeln von $\varphi(u)=0$ in $-\frac{\varphi^{\prime}(u)}{W^{2}}$ übergeht, so werden den einfachen Wurzeln von $\varphi(u)=0$ wirkliche Maxima oder Minima entsprechen. Die Differentiation von (6.) ergiebt

$$
\frac{2}{(u-v)^{2}}\left(\frac{d v}{d u}-1\right)=-\frac{1}{(u-a)^{2}}-\frac{1}{(u-b)^{2}}-\frac{1}{(u-c)^{2}}+\frac{1}{(u-\alpha)^{2}}+\frac{1}{(u-\beta)^{2}}
$$

setzt man hierin $\frac{d v}{d u}=0$ und eliminirt $v$ mit Hülfe von (6.), so kommt

$$
\left\{\begin{aligned}
2\left\{\frac{1}{(u-a)^{2}}+\frac{1}{(u-b)^{2}}+\frac{1}{(u-c)^{2}}-\frac{1}{(u-\alpha)^{2}}-\frac{1}{(u-\beta)^{2}}\right\} \\
-\left\{\frac{1}{u-a}+\frac{1}{u-b}+\frac{1}{u-c}-\frac{1}{u-\alpha}-\frac{1}{u-\beta}\right\}^{2}=0
\end{aligned}\right.
$$

welche Gleichung nach Wegschaffung der Nenner in $\varphi(u)=0$ übergeht.

Wir wollen jetzt die Anzahl und Grenzen der reellen Wurzeln dieser Gleichung achten Grades bestimmen.

\section{3 .}

Lemma. Es sei $\varphi(u)$ eine ganze Function von $u, \frac{d \varphi}{d u}=\varphi^{\prime}(u), \quad V$ und $\varphi^{\prime \prime}$ zwei beliebige aber durch die Relation

$$
\text { (11.) } \varphi-V \varphi^{\prime}+\varphi^{\prime \prime}=0
$$

von einander abhängige Functionen von $u$, so hat $\varphi(u)=0$ innerhalb jeden Intervalles von $u=A$ bis $u=B(A<B)$, in welchem $V$ und $\varphi^{\prime \prime}$ endlich bleiben und $\varphi^{\prime \prime}$ weder sein Zeichen wechselt noch verschwindet, erstens keine mehrfachen Wurzeln, und zweitens so viel reelle Wurzeln, als die Functionenreihe $\varphi, \varphi^{\prime}, \varphi^{\prime \prime}$ für $u=A$ mehr Zeichenwechsel hat, als für $u=B$, demnach höchstens zwei.

Der erste Theil der Behauptung ist evident; der zweite beweist sich nach der Sturmschen Methode, indem, wenn $u$ von $A$ bis $B$ wächst, Aenderungen in den Vorzeichen nur dadurch hervorgebracht werden, dass $u$ durch eine einfache Wurzel von $\varphi(u)=0$ oder $\varphi^{\prime}(u)=0$ geht, im ersten Falle aber be- 
116 Joachimsthal, Ansahl reeller Normalen von einem Punkte an ein Ellipsoid.

kanntlich ein Zeichenwechsel zwischen $\varphi$ und $\varphi^{\prime}$ verloren geht, im zweiten die Anzahl der Zeichenwechsel in der Reihe $\varphi, \varphi^{\prime} ; \varphi^{\prime \prime}$ ungeändert bleibt.

Diesen Hülfssatz wollen wir auf die Gleichung (10.) anwenden. Wir schreiben

$$
\begin{gathered}
(u-a)(u-b)(u-c)=D, \quad(u-\alpha)(u-\beta)=\Delta, \quad \frac{D}{\Delta}=z, \\
D^{2} \Delta^{2}\left\{\begin{array}{c}
2\left(\frac{1}{(u-a)^{2}}+\frac{1}{(u-b)^{2}}+\frac{1}{(u-c)^{2}}-\frac{1}{(u-\alpha)^{2}}-\frac{1}{(u-\beta)^{2}}\right) \\
-\left(\frac{1}{u-a}+\frac{1}{u-b}+\frac{1}{u-c}-\frac{1}{u-\alpha}-\frac{1}{u-\beta}\right)^{2}
\end{array}\right\},
\end{gathered}
$$

so ist

$$
\frac{z^{\prime}}{z}=\frac{1}{u-a}+\frac{1}{u-b}+\frac{1}{u-c}-\frac{1}{u-\alpha}-\frac{1}{u-\beta}
$$

und

$$
\varphi(u)=D^{2} \Delta^{2}\left\{\frac{2\left(z^{\prime 2}-z^{\prime \prime}\right)}{z^{2}}-\frac{z^{\prime 2}}{z^{2}}\right\}=\Delta^{4}\left(\dot{z}^{\prime 2}-2 z z^{\prime \prime}\right),
$$

woraus

und

$$
\varphi^{\prime}(u)=4 \Delta^{3} \Delta^{\prime}\left(z^{\prime 2}-2 z z^{\prime \prime}\right)-2 \Delta^{4} z z^{\prime \prime \prime}
$$

$$
4 \Delta^{\prime} \varphi(u)-\Delta \varphi^{\prime}(u)=2 \Delta^{4} D z^{\prime \prime \prime} .
$$

Nun ist

$$
z=\frac{D}{\Delta}=u+\lambda+\frac{(\alpha-a)(\alpha-b)(\alpha-c)}{\alpha-\beta} \frac{1}{u-\alpha}+\frac{(\beta-a)(\beta-b)(\beta-c)}{\beta-\alpha} \frac{1}{u-\beta},
$$

wo $\lambda$ kein $u$ enthält, folglich

$$
\Delta^{4} z^{\prime \prime \prime}=-6\left\{\frac{(\alpha-a)(\alpha-b)(\alpha-c)}{\alpha-\beta}(u-\beta)^{4}+\frac{(\beta-a)(\beta-b)(\beta-c)}{\beta-a}(u-\alpha)^{4}\right\}=-6 f(u) \text {. }
$$

Die Coefficienten von $(u-\beta)^{4}$ und $(u-\alpha)^{4}$ innerhalb der Klammer sind wesentlich negativ, also auch $f(u)$. Durch Substitution der Werthe von $\Delta^{\prime}, \Delta, D$ und $\Delta^{4} z^{\prime \prime \prime}$ verwandelt sich (12.) in die (11.) analoge Gleichung

$$
\varphi(u)-\frac{(u-\alpha)(u-\beta)}{4(2 u-\alpha-\beta)} \varphi^{\prime}(u)+\frac{3(u-a)(u-b)(u-c)}{2 u-\alpha-\beta} f(u)=0 .
$$

Bei der Anwendung des Lemma's können wir nach dem über $f(u)$ Gesagten

$$
\varphi^{\prime \prime}(u)=-\frac{(u-a)(u-b)(u-c)}{2 u-\alpha-\beta}
$$

setzen; eine Function, die ihr Zeichen ändert, sobald $u$ durch $a, b, c$ und $\frac{1}{2}(\alpha+\beta)$ geht. Nun ist $\frac{1}{2}(\alpha+\beta)<a$ und $>c$, kann aber eben sowohl $>b$ als $<b$ sein. Bei der Feststellung der Intervalle, in welchen $\varphi^{\prime \prime}$ sein Zeichen nicht ändert, mũssen diese beiden Fălle daher gesondert werden. 
Weil $\varphi(u)$ mit $+u^{8}$ beginnt, haben wir

(15.) $\left\{\begin{array}{l}\varphi(c)=(c-a)^{2}(c-b)^{2}(c-\alpha)^{2}(c-\beta)^{2} \text { also }=+ \text {, eben so } \varphi(b), \varphi(c), \\ \varphi(\beta)=-3(\beta-a)^{2}(\beta-b)^{2}(\beta-c)^{2}(\beta-\alpha)^{2} \text { also }=- \text {, ebenso } \varphi(\alpha) .\end{array}\right.$

Ferner

(16.)

$$
\left\{\begin{array}{l}
\varphi^{\prime}(-\infty)=-, \varphi^{\prime}(+\infty)=+, \text { und aus (13.) ergiebt sich } \\
\varphi^{\prime}(a)=\frac{4(2 a-\alpha-\beta)}{(a-\alpha)(a-\beta)} \varphi(a), \text { also }=+, \\
\varphi^{\prime}(b)=\frac{4(2 b-\alpha-\beta)}{(b-\alpha)(b-\beta)} \varphi(b), \text { also }=- \text {, wenn } b>\frac{1}{2}(\alpha+\beta), \\
\quad \text { und }=+ \text {, wenn } b<\frac{1}{2}(\alpha+\beta), \\
\varphi^{\prime}(c)=\frac{4(2 c-\alpha-\beta)}{(c-\alpha)(c-\beta)} \varphi(c) \text { also }=-, \\
\varphi^{\prime}\left(\frac{\alpha+\beta}{2}\right)=-\frac{48}{(\alpha-\beta)^{2}}\left(\frac{\alpha+\beta}{2}-a\right)\left(\frac{\alpha+\beta}{2}-b\right)\left(\frac{\alpha+\beta}{2}-c\right) f\left(\frac{\alpha+\beta}{2}\right), \\
\text { also }=+, \text { wenn } b>\frac{1}{2}(\alpha+\beta), \text { und }=-, \text { wenn } b<\frac{1}{2}(\alpha+\beta) .
\end{array}\right.
$$

Wir wollen den Raum von $-\infty$ bis $+\infty$ in kleinere Intervalle zerlegen, in welchen $\varphi^{\prime \prime}(u)$ jedesmal sein durch (14.) leicht zu bestimmendes Zeichen beibehält. Vorzeichen, die in (15.) und (16.) nicht bestimmt sind, haben wir in den nachfolgenden Tabellen durch ein Sternchen bezeichnet.

$$
\text { I. } b>\frac{1}{2}(\alpha+\beta)
$$

$$
\begin{aligned}
& u=\frac{1}{-\infty c-\varepsilon} \frac{2}{c+\varepsilon \beta} \frac{3}{\beta \frac{1}{2}(\alpha+\beta)-\varepsilon} \frac{4}{\frac{1}{2}(\alpha+\beta)+\varepsilon b-\varepsilon} \frac{5}{b+\varepsilon \alpha} \frac{6}{\alpha a-\varepsilon} \frac{7}{a+\varepsilon+\infty} \\
& \varphi=+++--\cdots \\
& \varphi^{\prime}=-\quad-\quad * *+ \\
& \varphi^{\prime \prime}=-\quad++++
\end{aligned}
$$

Nach dem Lemma ist also in den Intervallen (1.) und (7.) keine Wurzel, in den Intervallen (2.), (5.) und (6.) je eine, wie man auch die unbestimmt gelassenen Vorzeichen wählen mag; in (3.) höchstens eine, ebenso in (4.), also in dem Gesammtintervalle von $\beta$ bis $b$ (vorausgesetzt, dass $\frac{1}{2}(\alpha+\beta)$ selbst keine Wurzel ist) keine, eine oder zwei Wurzeln, da aber $\varphi(\beta)$ und $\varphi(b)$ entgegengesetzte Vorzeichen haben, so ist genau eine Wurzel vorhanden. Ist jedoch $\frac{1}{2}(\alpha+\beta)$ eine Wurzel, só kann es, weil $\varphi^{\prime}\left(\frac{\alpha+\beta}{2}\right)>0$, nur eine einfache sein. Dann gestaltet sich das Schema für beide Intervalle also: 
118 Joachimsthal, Anzahl reeller Normalen von einem Punkte an ein Ellipsoid.



Beide Intervalle enthalten demnach keine Wurzeln und das Gesammtintervall von $\beta$ bis $b$ nur eine Wurzel $=\frac{1}{2}(\alpha+\beta)$, wie oben.

II. $b<\frac{1}{2}(\alpha+\beta)$. Auch hier sind 7 Intervalle zu unterscheiden, von denen (1.), (2.), (6.), (7.) mit den obigen identisch sind; die drei übrigen sind

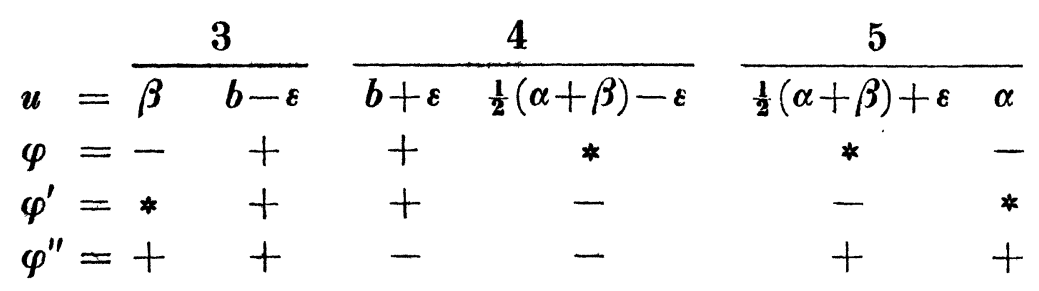

demnach ist in (3.) eine Wurzel, in (4.) + (5.) zusammen eine, und der Fall, dass $\varphi\left(\frac{\alpha+\beta}{2}\right)=0$, erledigt sich wie oben.

III. $b=\frac{1}{2}(\alpha+\beta) ; \varphi^{\prime \prime}(u)=-(u-a)(u-c)$ ist zwischen $a$ und $c$ positiv, sonst negativ; man hat die Intervalle $-\infty$ bis $c-\varepsilon, c+\varepsilon$ bis $b-\varepsilon$, $b+\varepsilon$ bis $a-\varepsilon, a+\varepsilon$ bis $+\infty$ zu unterscheiden, von denen (1.) und (4.) mit den früheren (1.) und (7.) identisch sind und keine Wurzeln enthalten, die beiden übrigen geben

$\begin{array}{lllll}\boldsymbol{u}=\boldsymbol{c}+\boldsymbol{\varepsilon} & \boldsymbol{b}-\boldsymbol{\varepsilon} & \boldsymbol{b}+\boldsymbol{\varepsilon} & \boldsymbol{a}-\boldsymbol{\varepsilon} \\ \boldsymbol{\varphi}= & + & + & + & + \\ \varphi^{\prime}= & - & * & * & + \\ \varphi^{\prime \prime}= & + & + & + & +\end{array}$

Ein jedes Intervall kann somit höchstens zwei Wurzeln enthalten, und enthält fie auch wirklich, weil $\varphi(c)=+, \varphi(\beta)=-, \varphi(b)=+, \varphi(\alpha)=-, \varphi(\alpha)=+$. Wir haben somit das Resultat:

Die Gleichung $\varphi(u)=0$ oder (10.) hat vier und nicht mehr reelle Wurzeln je ine zwischen $c$ und $\beta, \beta$ und $b, b$ und $\alpha, \alpha$ und $a$, und jede iderselben ist eine einfache.

Anmerk ung. Die Gleichung $\varphi(w)=0$ verdiente vielleicht auch wach anderen Bexiehungen eine genaute Discussion. Im ebenen Problem ist die 
entsprechende Gleichung

$$
2\left\{\frac{1}{(u-a)^{2}}+\frac{1}{(u-b)^{2}}-\frac{1}{(u-a)^{2}}\right\}-\left\{\frac{1}{u-a}+\frac{1}{u-b}-\frac{1}{u-\alpha}\right\}^{2}=0,
$$

für welche die obigen Resultate sich auf die verschiedenartigste Weise ableiten lassen, eine solche, deren Invariante zweiter Dimension (die Grösse $\boldsymbol{J}$ in der Note zu \$. 1) verschwindet. Drückt man dieselben durch die W.urzeln der biquadratischen Gleichung $\varepsilon_{1}, \varepsilon_{2}, \varepsilon_{3}, \varepsilon_{4}$ aus, so heisst dies $\Sigma\left(\varepsilon_{1}-\varepsilon_{2}\right)^{2}\left(\varepsilon_{3}-\varepsilon_{4}\right)^{2}=0$; eine Relation, die bei einer biquadratischen Gleichung mit reellen Coefficienten nur bestehen kann, wenn zwei der Wurzeln reell und zwei imaginär sind. Dieselbe Invariante verschwindet auch für die den vierten Grad nicht übersteigende Gleichung

$$
2\left(\frac{1}{(u-a)^{2}}+\frac{1}{(u-b)^{2}}+\frac{1}{(u-c)^{2}}-\frac{1}{(u-a)^{2}}\right)-\left(\frac{1}{u-a}+\frac{1}{u-b}+\frac{1}{u-c}-\frac{1}{u-a}\right)^{2}=0 .
$$

\$. 4.

Nennt man die Wurzeln von $\varphi(u)=0$ nach aufsteigender Grösse geordnet $u_{1}, u_{2}, u_{3}, u_{4}$ und die zugehőrigen durch die Gleichung

$$
\text { (6.) } \frac{2}{u-v}=\frac{1}{u-a}+\frac{1}{u-b}+\frac{1}{u-c}-\frac{1}{u-a}-\frac{1}{u-\beta}
$$

definirten Werthe von $v$ bezüglich $v_{1}, v_{2}, v_{3}, v_{4}$, so sind, da für $u=-\infty$, $v=+\infty$, beim Wachsen von $u$ also zuerst ein Abnehmen von $v$ stattfindet, $v_{1}$ und $v_{3}$ offenbar Minima, $v_{2}$ und $v_{4}$ Maxima, und man hat

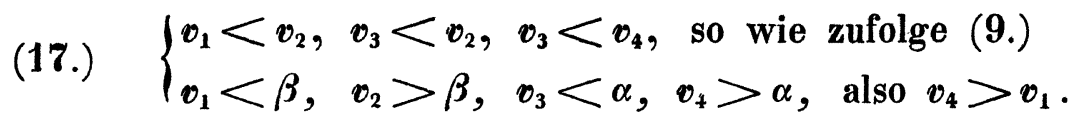

Trägt man auf der gegebenen Normale mit Berücksichtigung des Zeichens vom Fusspunkte $\left(x_{0}, y_{0}, z_{10}\right)$ aus die Strecken $\frac{\alpha}{\pi}, \frac{\beta}{\pi}, \frac{v_{1}}{\pi}, \frac{v_{2}}{\pi}, \frac{v_{3}}{\pi}, \frac{v_{4}}{\pi}$ ab (cf. \$. 1) und nennt die Endpunkte der Strecken $(\alpha),(\beta),\left(v_{1}\right)$ u. s. w., so haben nach (17.) diese Punkte folgende Lage:

$$
\begin{aligned}
& (-\infty)\left(v_{1}\right)(\beta)\left(v_{2}\right)(+\infty) ; \\
& (-\infty)\left(v_{3}\right)(\alpha)\left(v_{4}\right)+\infty ; \\
& (-\infty)\left(v_{3}\right)\left(v_{2}\right)(+\infty) ; \\
& (-\infty)\left(v_{1}\right)\left(v_{4}\right)(+\infty)
\end{aligned}
$$

Namentlich aber können die Punkte $\left(v_{1}\right),\left(v_{2}\right),\left(v_{3}\right),\left(v_{4}\right)$, welche die nach beiden Seiten unendliche Normate in funf Stukcke zerlegen, nur eine der fol- 
genden vier Lagen haben:

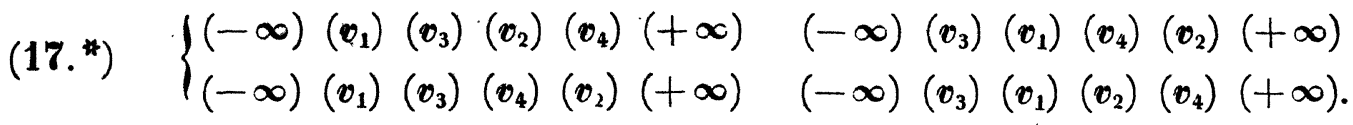

Die Strecken $\overline{\left(v_{1}\right)\left(v_{2}\right)}$ und $\overline{\left(v_{3}\right)\left(v_{4}\right)}$ fallen also entweder zum Theil auf einander, oder die eine liegt in der anderen.

Während $u$ von $-\infty$ bis $+\infty$ wächst, geht $v$ continuirlich von $+\infty$ bis $v_{1}$, von $v_{1}$ nach $v_{2}$, von $v_{2}$ nach $v_{3}$, von $v_{3}$ nach $v_{4}$, von $v_{4}$ nach $-\infty$. Verfolgt man diese Bewegung in jeder der obigen vier Anordnungen (17.*), so sieht man, dass der Endpunkt (v) der Strecke $\frac{v}{\pi}$ jedesmal die beiden äussersten (nur nach einer Seite begrenzten) Theile der Normale einmal, die ihnen angrenzenden dreimal, den mittelsten fünfmal durchläuft.

Uebertragen wir dies auf die vorliegende Aufgabe, so heisst dies: von einem Punkte $(\xi, \eta, \zeta)$ der gegebenen Normale lassen sich noch fünf, drei oder eine Normale an das Ellipsoid legen, je nachdem der Punkt $(\xi, \eta, \zeta)$ auf beiden Strecken $\overline{\left(v_{1}\right)\left(v_{2}\right)}, \overline{\left(v_{3}\right)\left(v_{4}\right)}$, oder nur auf einer, oder auf keiner liegt.

Mit Hülfe einiger das Ellipsoid betreffende Formeln lässt sich dieses Resultat einfach ausdrücken; wir wollen dieselben jetzt zusammenstellen.

\section{\$. 5 .}

Sind $\alpha$ und $\beta$ die Quadrate der Halbaxen des der Tangentialebene im Punkte $\left(x_{10}, y_{0}, z_{0}\right)$ des Ellipsoides parallelen Diametralschnittes, so ist

$$
\left(4 .^{*}\right) \quad x_{0}^{2}=\frac{a(a-\alpha)(a-\beta)}{(a-b)(a-c)}, \quad y_{0}^{2}=\frac{b(b-\alpha)(b-\beta)}{(b-a)(b-c)}, \quad z_{11}^{2}=\frac{c(c-\alpha)(c-\beta)}{(c-a)(c-b)} .
$$

Die Hauptkrümmungsradien sind $\frac{\alpha}{\pi}$ und $\frac{\beta}{\pi}$ (und beiläufig $\pi^{2} \alpha \beta=a b c$ ). Ist $(\boldsymbol{X}, \boldsymbol{Y}, \boldsymbol{Z})$ der zugehörige Krümmungsmittelpunkt, so hat man:

$$
\frac{X-x_{0}}{\frac{x_{0}}{a}}=\frac{Y-y_{0}}{\frac{y_{0}}{b}}=\frac{Z-z_{0}}{\frac{z_{0}}{c}}=-\alpha \text { oder }=-\beta ; \text { und hieraus }
$$

(18.) $\quad X^{2}=\frac{(a-u)^{3}(a-w)}{a(a-b)(a-c)}, \quad Y^{2}=\frac{(b-u)^{3}(b-w)}{b(b-a)(b-c)}, \quad Z^{2}=\frac{(c-u)^{3}(c-w)}{c(c-a)(c-b)}$,

wo entweder $u=\alpha, w=\beta$ oder $u=\beta, w=\alpha$ zu setzen ist. Giebt man aber $u$ alle möglichen Werthe von $a$ bis $b$, und $w$ gleichzeitig alle Werthe von $b$ bis $c$, so ist der Ort des Punktes $\left(X, Y, Z\right.$ ) eine F́läche $F_{1}$ (der Ort der Mittelpunkte der grösseren Hauptkrümmungskreise), und wenn man die Inter- 
valle für $u$ und $v$ vertauscht, so ist der Ort eine zweite Fläche $F_{2}$, welche die Centra der kleinen Hauptkrümmungskreise enthält; $F_{1}$ und $F_{2}$ können als Theile einer Fläche angesehen werden, deren Gleichung durch Elimination von $u$ und $w$ aus (18.) erhalten wird, die Fläche der Krümmungsmittelpunkte. Jede durch den Anfangspunkt gezogene Grade trifft $F_{1}$ in zwei zu diesem symmetrisch gelegenen Punkten und eben so $F_{2}$. Denn soll $X: Y: Z=l: m: n$ sein, so sind $u$ und $w$ durch die Gleichung bestimmt:

$$
l^{2}: m^{2}: n^{2}=\frac{(a-u)^{3}(a-w)}{a(a-b)(a-c)}: \frac{(b-u)^{3}(b-w)}{b(b-a)(b-c)}: \frac{(c-u)^{3}(c-w)}{c(c-a)(c-b)}
$$

woraus

$$
\text { (20.) } \quad \frac{a l^{2}}{(a-u)^{3}}+\frac{b m^{2}}{(b-u)^{3}}+\frac{c n^{2}}{(c-u)^{3}}=0,
$$

welche Gleichung nach bekannter Schlussfolge nur zwei reelle Wurzeln, eine zwischen $a$ und $b$, und eine zweite zwischen $b$ und $c$ 'enthält. Aus (19.) folgt für jedes reelle $u$ zwischen $a$ und $b$ (b und $c$ ) ein reelles $w$ zwischen $b$ und $c\left(a\right.$ und $b$ ). Demnach sind $F_{1}$ und $F_{2}$ geschlossene Flächen.

Wir wollen jetzt die Durchschnitte der Normale des Ellipsoids am Punkte $\left(x_{1}, y_{0}, z_{0}\right)$ mit den Flächen $F_{1}$ und $F_{2}$ bestimmen. Es seien $X, Y, Z$ die Coordinaten eines Durchschnittes, so müssen ausser (18.) noch die Gleichungen

$$
\frac{X-x_{0}}{\frac{x_{0}}{a}}=\frac{Y-y_{0}}{\frac{y_{0}}{b}}=\frac{Z-z_{0}}{\frac{z_{0}}{c}}=-v
$$

stattfinden. Substituirt man die hieraus sich ergebenden Werthe von $X, Y, Z$ in (18.), so werden $u, w, v$ durch die Gleichungen bestimmt:

$$
\left\{\begin{array}{l}
(a-u)^{3}(a-v)=(a-v)^{2}(a-\alpha)(a-\beta), \\
(b-u)^{3}(b-v)=(b-v)^{2}(b-\alpha)(b-\beta), \\
(c-u)^{3}(c-v)=(c-v)^{2}(c-\alpha)(c-\beta) .
\end{array}\right.
$$

Die Añnahme $u=a$ giebt $v=a$, und aus den beiden anderen Gleichungen in (21.)

$$
-w=a-\alpha-\beta+\frac{(a-\alpha)(a-\beta)}{b-a}, \quad-w=a-\alpha-\beta+\frac{(a-\alpha)(a-\beta)}{c-a},
$$

was, weil $a-\alpha, a-\beta$ von Null verschieden sind, auf einen Widerspruch führt; eben so wenig kann $u=b$ oder $=c$ sein. Wir können demnach aus (21.) die beiden Gleichungen ableiten : 
(22.)

$$
\begin{aligned}
& \text { (22.) } \frac{(a-v)^{2}}{(a-u)^{2}} \frac{(a-\alpha)(a-\beta)}{(a-b)(a-c)}+\frac{(b-v)^{2}}{(b-u)^{2}} \frac{(b-\alpha)(b-\beta)}{(b-a)(b-c)}+\frac{(c-v)^{2}}{(c-u)^{2}} \frac{(c-a)(c-\beta)}{(c-a)(c-b)}-1=0, \\
& \text { (23.) } \frac{(a-v)^{2}}{(a-u-\alpha)(a-\beta)} \frac{(a-\beta)}{(a-b)(a-c)}+\frac{(b-v)^{2}}{(b-u)^{3}} \frac{(b-\alpha)(b-\beta)}{(b-a)(b-c)}+\frac{(c-v)^{2}}{(c-u-\alpha)(c-\beta)}=0 .
\end{aligned}
$$

Umgekehrt lassen sich aus den beiden Gleichungen (22.) und (23.) die drei Gleichungen (21.) ableiten; denn drei beliebige Grössen $\gamma, \gamma^{\prime}, \gamma^{\prime \prime}$ können, wenn $(a-b)(a-c)(b-c) \gtrless 0$, immer unter die Form gebracht werden:

$$
\gamma=\varepsilon+\varepsilon^{\prime} a+\varepsilon^{\prime \prime} a^{2}, \quad \gamma^{\prime}=\varepsilon+\varepsilon^{\prime} b+\varepsilon^{\prime \prime} b^{2}, \quad \gamma^{\prime \prime}=\varepsilon+\varepsilon^{\prime} c+\varepsilon^{\prime \prime} c^{2} .
$$

Schreibt man statt (22.) und (23.)

und

$$
\frac{\gamma}{(a-b)(a-c)}+\frac{\gamma^{\prime}}{(b-a)(b-c)}+\frac{\gamma^{\prime \prime}}{(c-a)(c-b)}=1
$$

$$
\frac{\gamma}{(a-b)(a-c)} \frac{1}{a-u}+\frac{\gamma^{\prime}}{(b-a)(b-c)} \frac{1}{b-u}+\frac{\gamma^{\prime \prime}}{(c-a)(c-b)} \frac{1}{c-u}=0,
$$

und substituirt für $\gamma \varepsilon+\varepsilon^{\prime} a+\varepsilon^{\prime \prime} a^{2}$ u. s. w., so ergiebt sich erstens, dass $\varepsilon^{\prime \prime}=1$, und zweitens, dass $\varepsilon+\varepsilon^{\prime} t+t^{2}$ den Factor $t-u$ enthält, also gleich $(t-u)(t-w)$ gesetzt werden kann; demnach ist $\gamma=(a-u)(a-w)$ u. s. w. Erwägt man, dass, wenn $z_{1}$ die ganze Function $F(z)$ nicht verschwinden lässt, $z_{1}$ eine doppelte Wurzel von $f(z)=0$ ist, sobald es den Gleichungen $\frac{f(z)}{F(z)}=0$, $\frac{d \frac{f(z)}{F(z)}}{d z}=0$

genügt, so sieht man, dass für $v$ nur solche Werthe zu nehmen sind, die der Gleichung (22.) eine Doppelwurzel verschaffen, und für $u$ diese Wurzel selbst, mit Ausschluss von $u=a,=b$ und $=c$. Nun ist Gleichung (22.) keine andere als (5.); und nach den Rechnungen in $\$ .1$ und $\$ .2$ kann man für dieselbe schreiben:

$$
\frac{(u-v)^{2}(u-\alpha)(u-\beta)}{(u-a)(u-b)(u-c)}\left\{\frac{1}{u-a}+\frac{1}{u-b}+\frac{1}{u-c}-\frac{1}{u-\alpha}-\frac{1}{u-\beta}-\frac{2}{u-v}\right\}=0
$$

oder

$$
\frac{(u-v)}{(u-a)^{2}(u-b)^{2}(u-c)^{2}}\{W(u-v)-2(u-a)(u-b)(u-c)(u-\alpha)(u-\beta)\}=0 .
$$

Die Werthe von $v$, welche dieser Gleichung eine Doppelwurzel verschaffen, machen entweder $u=0$, und dann sind sie (wiederum mit Ausschluss von $a, b$ und $c)=\alpha$ und $=\beta$, oder sie geben dem anderen Factor eine Doppelwurzel, und dann hat man für diese 
Joachimsthal, Anzahl reeller Normalen von einem Punkte an ein Ellipsoid. 123

$$
\begin{aligned}
& \frac{1}{u-a}+\frac{1}{u-b}+\frac{1}{u-c}-\frac{1}{u-\alpha}-\frac{1}{u-\beta}-\frac{2}{u-v}=0 \\
& \frac{1}{(u-a)^{2}}+\frac{1}{(u-b)^{2}}+\frac{1}{(u-c)^{2}}-\frac{1}{(u-\alpha)^{2}}-\frac{1}{(u-\beta)^{2}}-\frac{2}{(u-v)^{2}}=0
\end{aligned}
$$

man kommt also auf die behandelte Gleichung (10.) wieder zurück* ${ }^{*}$ ). Somit haben die Gleichungen (22.) und (23.) nur folgende sechs Lösungen:

$$
\begin{array}{lllllll}
v & =\beta & v_{1} & v_{2} & \alpha & v_{3} & v_{4} \\
u=\beta & u_{1} & u_{2} & \alpha & u_{3} & u_{4} .
\end{array}
$$

$\mathrm{Zu}$ jedem Werthepaare von $v$ und $u$ bestimmt sich aus (21.) ein reelles $w$, das zwischen $a$ und $b$ liegt, wenn $u$ zwischen $b$ und $c$ liegt und umgekehrt. Die Normale am Punkte $\left(x_{10}, y_{10}, z_{11}\right)$ des Ellipsoides hat also mit der Fläche $F_{1}$ die drei in $\$ \$ .4$ mit $(\alpha),\left(v_{3}\right),\left(v_{4}\right)$ bezeichneten Punkte gemeinschaftlich, und mit $F_{2}$ die Punkte $(\beta),\left(v_{1}\right),\left(v_{2}\right)$. Dass die Normale mit jeder der geschlossenen Flächen $F_{1}, F_{2}$ eine ungrade Anzahl von Punkten gemeinschaftlich hat, erklärt sich daraus, dass sie $F_{1}$ in $(\alpha), F_{2}$ in $(\beta)$ berührt. Wenn man den Beweis dafür analytisch führen will, so kann man entweder auf die Formeln (18.) zurückgehen, oder es unmittelbar daraus schliessen, dass die Discriminante von (5.) oder von

$$
(u-v)\{W(u-v)-2(u-a)(u-b)(u-c)(u-\alpha)(u-\beta)\}=v
$$

offenbar die quadratischen Factoren $(v-\alpha)^{2},(v-\beta)^{2}$ enthält. Da für die vorliegende Untersuchung dieser Umstand aber ohne Bedeutung ist, so gehen wir auf diesen Beweis nicht näher ein.

Die Resultate des \$.,4 lassen sich demnach folgendermaassen aussprechen:

Eine nicht in einem Hauptschnitte des Ellipsoides liegende Normale trifft jeden der beiden Theile $F_{1}, F_{2}$ der Fläche der Krümmungsmittelpunkte, abgesehen von den beiden Krümmungsmittelpunkten $(\alpha)$ und $(\beta)$, welche zum Fusspunkte der Normalen gehören, in noch zwei Punkten $\left(v_{3}\right),\left(v_{4}\right)$ und $\left(v_{1}\right),\left(v_{2}\right)$. Die beiden von den Normalen in $F_{1}$ und $F_{2}$ bestimmten Sehnen enthalten jede eines der Centra $(\alpha),(\beta)$ und sind durch keinen endlichen Raum von einander getrennt, d. h. die eine liegt zum Theil oder ganz in der anderen. Ferner: Von einem Punkte lassen sich an ein Ellipsoid sechs, vier oder zwei Normalen ziehen, je nachdem er innerhalb beider Theile $F_{1}$ und $F_{2}$ der Fläche

*) Man hätte diese Betrachtungen mit Hülfe der Curve, welche (22.) oder (5.) repräsentirt und die aus einer parabolischen Curve fünften Grades und einer Graden besteht, wenn $u$ und $v$ als Coordinaten angesehen werden, abkürzen können. 
der Krümmungsmittelpunkte, oder innerhalb des einen und ausserhalb des anderen, oder ausserhalb beider liegt.

Anmerkung. Zum vollständigen Beweise dieses Resultates gehört noch die Betrachtung des Falles, dass der gegebene Punkt in einer Hauptebene liegt.

Die $(x y)$ Ebene schneidet die beiden Flächen $F_{1}$ und $F_{2}$ in der Curve

$$
\begin{array}{rlrl}
\text { (24.) } & \left(a x^{2}\right)^{\frac{1}{3}}+\left(b y^{2}\right)^{\frac{1}{3}} & =(a-b)^{\frac{1}{3}}, \\
\text { (25.) } & \frac{a x^{2}}{(a-c)^{2}}+\frac{b y^{2}}{(b-c)^{2}}=1
\end{array}
$$

Von den Fusspunkten der sechs durch $(\xi \eta)$ gehenden Normalen liegen vier in der $(x y)$ Ebene, und ihre Realität hängt davon ab, ob $(\xi \eta)$ innerhalb (24.) liegt oder nicht; die beiden anderen ausserhalb der $(x y)$ Ebene, und ihre Realität hängt davon $\mathrm{ab}$, ob $(\xi \eta)$ innerhalb (25.) liegt oder nicht. Die Combination beider Resultate zeigt die Richtigkeit des Hauptsatzes für diesen speciellen Fall.

Von den beiden Curven, der Ellipsenevolute und einer Ellipse, aus welchen die Durchschnitte von $F_{1}$ und $F_{2}$ mit den Coordinatenebenen bestehen, gehört zu $F_{1}$ in dem einen Hauptschnitte die Evolute, in dem anderen die Ellipse, in dem dritten ein Theil der Evoluten und ein Theil der Ellipse, ebenso ist es mit $\boldsymbol{F}_{2}$.

\section{Breslau, im Januar $\left.1861^{*}\right)$.}

*) Noch ehe diese Abhandlung in die Oeffentlichkeit gelangt ist, hat ein frühzeitiger Tod am 5. d. M. ihren scharfsinnigen Verfasser aus der Mitte seiner Laufbahn fortgerafft.

Die Grösse des Verlustes, den die Wissenschaft durch seinen Tod erleidet, wissen die Leser dieser Zeitschrift zu ermessen, die ihn aus einer Reihe von Abhandlungen als vielseitigen Forscher in den verschiedensten Theilen der Analysis und vor Allem in ihrer Anwendung auf die Geometrie kennen. Er begnügte sich nicht, die Fragen, die er behandelte, durch Hinzufügung einzelner neuer Thatsachen und Ergebnisse zu fördern, sondern ging überall bis zu den einfachsten Gründen zurück und suchte so zwischen vereinzelten Ergebnissen einen Zusammenhang aufzufinden. In dieser auf das Ganze gerichteten Thätigkeit seines klaren Geistes lag die seltene Lehrergabe, die ihn auszeichnete, und die seinen Tod für den mathematischen Unterricht zu einem ebenso beklagenswerthen Ereigniss macht, wie für die mathematische Literatur.

Berlin, im April 1861.

B. 